doi: $10.2306 /$ scienceasia1513-1874.2013.39.561

\title{
Maximum likelihood estimation of linear structural relationship model parameters assuming the slope is known
}

\author{
Abu S.M.A. Mamun ${ }^{\mathrm{a}, *}$, Ghapor A. Hussin ${ }^{\mathrm{b}}$, Yong Z. Zubairi ${ }^{\mathrm{a}}$, Rahmatullah A.H.M. Imon ${ }^{\mathrm{c}}$ \\ a Mathematics Division, Centre for Foundation Studies in Science, University of Malaya, \\ 50603 Kuala Lumpur, Malaysia \\ b Faculty of Science and Defence Technology, National Defence University of Malaysia, Kuala Lumpur, \\ Malaysia \\ c Department of Mathematical Sciences, Ball State University, Muncie, IN 47306, USA
}

${ }^{*}$ Corresponding author, e-mail: mithun_stat@yahoo.com

Received 7 Oct 2012

Accepted 16 Aug 2013

\begin{abstract}
A number of statistical techniques have been proposed by many authors to estimate the parameters in a linear structural relationship model, but only few papers discuss the precision of these estimators. In this study, we derive the maximum likelihood estimate (MLE) of the parameters by assuming the slope parameter $\beta$ is known. $\beta$ is estimated separately by a nonparametric method and is assumed to be known when other parameters are estimated by an MLE. We obtain closed-form estimates of parameters as well as the variance-covariance matrix. Using a simulation study and a real-world example we show that the estimated values of the parameters are unbiased and consistent.
\end{abstract}

KEYWORDS: errors-in-variables model, nonparametric method, asymptotic variance-covariance matrix

\section{INTRODUCTION}

An errors-in-variables model (EIVM) is a study of relationships between variables, groups or populations in which errors are present in both the explanatory and response variables. It differs from the traditional regression where errors are present only in the dependent variable. Because of the measurement error in explanatory variables, the traditional regression often produces biased estimates of parameters. The linear structural relationship model (LSRM) is one of the families in the EIVM which also includes functional, ultrafunctional, and ultrastructural relationship models. Many authors have considered EIVM over the years ${ }^{1-4}$. Although a lot of work has been done on the LSRM, only a few studies focused on the precision of the parameter estimation. Bolfarine and Cordani ${ }^{5}$, Patefield ${ }^{6}$ and Wong ${ }^{7}$ considered only a few special cases such as the accuracy of the slope and intercept. Hood et $\mathrm{al}^{8}$ first described the precision of the parameters and established the asymptotic variancecovariance matrix when the ratio of the two error variances is known. As for the estimation of slope parameter, several methods of estimation are proposed such as geometric mean ${ }^{9}$, by assuming equal error variance ${ }^{10}$, repeated median ${ }^{11}$, and nonparametric $\operatorname{method}^{12}$.

As far as we know, there has been no attempt to estimate parameters assuming that the slope parameter $\beta$ is known. Once we assume that $\beta$ is known, we can easily estimate the other parameters by the maximum likelihood method. But the main advantage of our method is that, unlike other methods, $\beta$ is not an arbitrary choice. In this article, we extend the idea of Al-Nasser ${ }^{12}$ to estimate the slope parameter by a nonparametric method and then estimate the rest of LSRM parameters of by maximum likelihood estimation (MLE). The precision of the estimated parameters and its asymptotic variance-covariance matrix are also derived and investigated here through real world data and simulations.

The applications of EIVMs can be seen in studies in the life sciences such as biology, ecology, social science and economics where the variables cannot be recorded accurately. Since our proposed method of estimation is an improvement over the existing ones it should have wider applications in the above areas of research. This paper is organized in the following way. First, we briefly review different aspects of LSRM. Next, we propose a nonparametric method to estimate the slope parameter extending the work of AlNasser ${ }^{12}$. The parameter estimation of LSRM by the 
MLE method assuming the slope is given is proposed in the next section. Then we derive the asymptotic closed form of the variance-covariance information matrix. We also report on simulation studies which are designed to investigate the precision of the estimated parameters and its variance-covariance matrix. A real world example is presented finally to show the advantage of the proposed method over the existing ones.

\section{LINEAR STRUCTURAL RELATIONSHIP MODEL}

Consider the model

$$
Y=\alpha+\beta X
$$

where there exists a linear relationship between the random variables $X$ and $Y$ and suppose that they are measured without error. However, in reality they are subject to error. Assume that for each $i=1,2, \ldots, n$, $x_{i}$ and $y_{i}$ are taken instead of $X_{i}$ and $Y_{i}$, respectively. If $\delta_{i}$ and $\epsilon_{i}$ are the two respective errors in measuring $X_{i}$ and $Y_{i}$, then we can write

$$
x_{i}=X_{i}+\delta_{i}, \quad y_{i}=Y_{i}+\epsilon_{i},
$$

where the error terms $\delta_{i}$ and $\epsilon_{i}$ are normally distributed having zero mean and variance $\sigma_{\delta}^{2}$ and $\sigma_{\epsilon}^{2}$, respectively. This reveals that the variances of error are not dependent on $i$ and so independent of the level of $X$ and $Y$. This property is known as homoscedasticity. There are some assumptions that have been described in the literature for obtaining the $X$ values. For example, Kendall and Stuart ${ }^{13}$ described the structural model considering $X_{i}$ as normal with mean $\mu$ and variance $\sigma_{X}^{2}$. In LSRMs, the errors are assumed to be normal and the bivariate normal distribution of $x_{i}$ and $y_{i}$ is then

$$
\left(\begin{array}{c}
x_{i} \\
y_{i}
\end{array}\right) \sim N\left(\left(\begin{array}{c}
\mu \\
\alpha+\beta \mu
\end{array}\right),\left(\begin{array}{cc}
\sigma_{X}^{2}+\sigma_{\delta}^{2} & \beta \sigma_{X}^{2} \\
\beta \sigma_{X}^{2} & \beta^{2} \sigma_{X}^{2}+\sigma_{\epsilon}^{2}
\end{array}\right)\right) \text {. }
$$

Kendall and Stuart ${ }^{13}$ stated the moment equations for the parameters of LSRM in the form

$$
\begin{gathered}
\mu=\bar{x}, \quad \beta \mu+\alpha=\bar{y}, \\
\sigma_{X}^{2}+\sigma_{\delta}^{2}=S_{x}^{2}, \quad \beta^{2} \sigma_{X}^{2}+\sigma_{\epsilon}^{2}=S_{y}^{2}, \quad \beta \sigma_{X}^{2}=S_{x y},
\end{gathered}
$$

where $S_{x}^{2}=(1 / n) \sum\left(x_{i}-\bar{x}\right)^{2}, S_{y}^{2}=(1 / n) \sum\left(y_{i}-\right.$ $\bar{y})^{2}$, and $S_{x y}=(1 / n) \sum\left(x_{i}-\bar{x}\right) \sum\left(y_{i}-\bar{y}\right)$. Clearly there are five equations in six unknowns, so some additional assumptions have to be made about the parameters of the LSRM in order to overcome this unidentifiability problem. Hood et $\mathrm{al}^{8}$ considered the following five cases for unique and consistent solutions to the equations. (i) both the error variances $\sigma_{\delta}^{2}$ and $\sigma_{\epsilon}^{2}$ are known;

(ii) $\sigma_{\delta}^{2}$ is known;

(iii) $\sigma_{\epsilon}^{2}$ is known;

(iv) both the error variances $\sigma_{\delta}^{2}$ and $\sigma_{\epsilon}^{2}$ are unknown;

(v) the error variances ratio $\lambda=\sigma_{\epsilon}^{2} / \sigma_{\delta}^{2}$ is known;

In this article, we develop another new case to solve the unidentifiability problem of estimating parameters in LSRMs. We first estimate the slope parameter by modifying the nonparametric method and then the rest of the parameters of the LSRM are estimated by the maximum likelihood method.

\section{ESTIMATION OF SLOPE PARAMETER BY USING NONPARAMETRIC METHOD}

In this method, the observed pairs $\left(x_{i}, y_{i}\right),(i=$ $1,2, \ldots, n)$ are ordered such that $x_{i}<x_{i+1}$ assuming all the $x_{i}$ are distinct. Then we distribute the observations into several groups to calculate all possible paired slopes. Furthermore, another possible pair of slopes is determined by ordering the observed pairs according to the magnitude of the $y$ value. The complete procedure is summarized in the following algorithm.

\section{Algorithm 1}

Step 1: We arrange the observations in ascending order on the magnitude of $x$ i.e., $x_{(1)} \leqslant x_{(2)} \leqslant$ $, \ldots, \leqslant x_{(n)}$. We take the associated values of $y$, i.e., $y_{[1]}, y_{[2]}, \ldots, y_{[n]}$ which may not be in ascending order and obtain our desire pairs $\left(x_{(i)}, y_{[i]}\right)$.

Step 2: We distribute the $n$ observations into $m$ subsamples each having $r$ elements such that $m r=$ $n$.

Step 3: Find the number of all possible combination of paired slopes $b(k)_{i j}=\left(y_{[j]}-y_{[i]}\right) /\left(x_{(j)}-\right.$ $\left.x_{(i)}\right)$, where $i=1,2, \ldots, j-1 ; j=2,3, \ldots, r$; and $k=1,2, \ldots, m$.

Step 4: Calculate $\beta_{x}=\operatorname{median}_{k} b(k)_{i j}$.

Step 5: We repeat Steps 1-4 by interchanging $y$ and $x$. Finally, we define the estimated value of the slope as $\hat{\beta}_{\text {new }}=\frac{1}{2}\left(\beta_{x}+\beta_{y}\right)$.

Steps 1-4 have been proposed by Al-Nasser ${ }^{12}$ and to improve the estimate we propose Step 5 by virtue of the symmetrical nature of variables $X$ and $Y$ in EIVM.

\section{MLE OF A LSRM ASSUMING KNOWN SLOPE}

Once the slope is estimated using the nonparametric method as described in the previous section, the other parameters can be estimated using the maximum likelihood method for the given value of the slope parameter. In this section, we briefly describe the procedure 
of deriving the estimating equation for the LSRM by using MLE. One advantage of this approach is that it is easy to get the asymptotic variance-covariance matrix of the estimators. Let

$$
A=S_{x}^{2}\left(\beta^{2} \sigma_{X}^{2}+\sigma_{\epsilon}^{2}\right)-2 S_{x y} \beta \sigma_{X}^{2}+S_{y}^{2}\left(\sigma_{X}^{2}+\sigma_{\delta}^{2}\right),
$$

the determinant of the variance-covariance matrix is

$$
B=\beta^{2} \sigma_{X}^{2} \sigma_{\delta}^{2}+\sigma_{\delta}^{2} \sigma_{\epsilon}^{2}+\sigma_{X}^{2} \sigma_{\epsilon}^{2}
$$

and

$$
\begin{gathered}
C=(\bar{x}-\mu)^{2}\left(\beta^{2} \sigma_{X}^{2}+\sigma_{\epsilon}^{2}\right)-2 \beta \sigma_{X}^{2}(\bar{x}-\mu) \\
(\bar{y}-\alpha-\beta \mu)+(\bar{y}-\alpha-\beta \mu)^{2}\left(\sigma_{X}^{2}+\sigma_{\delta}^{2}\right) .
\end{gathered}
$$

The log-likelihood function can be rewritten in the following way

$$
l=-n \log (2 \pi)-\frac{n}{2}\left\{\frac{A}{B}+\frac{C}{B}+\log (B)\right\} .
$$

The term $C$ in log-likelihood function will be minimized, and equal to zero, when $\mu=\bar{x}$ and $\beta \mu+\alpha=\bar{y}$. From this equation, maximum likelihood estimates of $\mu$ and $\alpha$ can be determined. Since the terms $A$ and $B$ in the log-likelihood function do not contain the parameters $\mu$ and $\alpha$, the likelihood function is then solved by minimizing

$$
G=-\frac{n}{2} \log (B)-\frac{n}{2}\left(\frac{A}{B}\right)
$$

with respect to $\sigma_{X}^{2}, \sigma_{\delta}^{2}$ and $\sigma_{\epsilon}^{2}$. The partial derivatives are taken and set equal to zero to give

$$
\begin{gathered}
\left(\beta^{2} \sigma_{\delta}^{2}+\sigma_{\epsilon}^{2}+\beta^{2} S_{X}^{2}-2 \beta S_{x y}+S_{y}^{2}\right) B \\
-\left(\beta^{2} \sigma_{\delta}^{2}+\sigma_{\epsilon}^{2} A\right)=0 \\
\left(\sigma_{\epsilon}^{2}+\beta^{2} \sigma_{X}^{2}+S_{y}^{2}\right) B-\left(\sigma_{\epsilon}^{2}+\beta^{2} \sigma_{X}^{2}\right) A=0 \\
\left(\sigma_{\delta}^{2}+\sigma_{X}^{2}+S_{x}^{2}\right) B-\left(\sigma_{\delta}^{2}+\sigma_{X}^{2}\right) A=0 .
\end{gathered}
$$

We solve the above three equations and get

$$
\begin{aligned}
\sigma_{X}^{2}=\frac{S_{x y}}{\beta}, \quad \sigma_{\delta}^{2} & =\frac{\left(\beta S_{x}^{2}-S_{x y}\right)}{\beta}, \\
\sigma_{\epsilon}^{2} & =\left(S_{y}^{2}-\beta S_{x y}\right) .
\end{aligned}
$$

Admissible solutions are obtained when $\beta S_{x}^{2}>S_{x y}$ and $S_{y}^{2}>\beta S_{x y}$. For some specific data set, if these criteria are not satisfied, we then set one or more variances equal to zero in order to find the maximum likelihood estimators for the parameters. Hence, if the first and second conditions are broken then following Ref. 8, we set $\sigma_{\delta}^{2}=0$ and $\sigma_{\epsilon}^{2}=0$.

\section{VARIANCE-COVARIANCE MATRIX OF A LSRM}

The Fisher information matrix $\left(I_{i j}\right)$ for the set of unknown parameters $\theta$ is given by

$$
\left(I_{i j}\right)=\left[-E\left(\frac{\partial^{2} l}{\partial \theta_{i} \partial \theta_{j}}\right)\right] \text {. }
$$

The above information matrix is inverted in order to find the asymptotic variance-covariance matrix. From (2),

$$
l=-n \log (2 \pi)-\frac{n}{2} \log (B)-\frac{D}{2 B}
$$

where

$$
\begin{gathered}
D=\left(\beta^{2} \sigma_{X}^{2}+\sigma_{\epsilon}^{2}\right) \sum\left(x_{i}-\mu\right)^{2}-2 \beta \sigma_{X}^{2} \sum\left(x_{i}-\mu\right) \\
\left(y_{i}-\alpha-\beta \mu\right)+\left(\sigma_{X}^{2}+\sigma_{\delta}^{2}\right) \sum\left(y_{i}-\alpha-\beta \mu\right)^{2}
\end{gathered}
$$

After lengthy algebraic manipulations, the inverse of the Fisher information matrix is given by

$$
\frac{1}{n}\left(\begin{array}{ccccc}
a_{11} & a_{12} & a_{13} & a_{14} & a_{15} \\
& a_{22} & a_{23} & a_{24} & a_{25} \\
& & a_{33} & a_{34} & a_{35} \\
& & & a_{44} & a_{45} \\
& & & & a_{55}
\end{array}\right)
$$

where $a_{11}=\sigma_{X}^{2}+\sigma_{\delta}^{2}, a_{12}=-\beta \sigma_{\delta}^{2}, a_{13}=0, a_{14}=$ $0, a_{15}=0, a_{22}=\beta^{2} \sigma_{\delta}^{2}+\sigma_{\epsilon}^{2}, a_{23}=0, a_{24}=0, a_{25}=$ $0, a_{33}=2 \sigma_{X}^{4}+B / \beta^{2}, a_{34}=2 \sigma_{\delta}^{2} \sigma_{X}^{2}-B / \beta^{2}, a_{35}=$ $2 \sigma_{\epsilon}^{2} \sigma_{X}^{2}-B, a_{44}=2 \sigma_{\delta}^{4}+B / \beta^{2}, a_{45}=2 \sigma_{\epsilon}^{2} \sigma_{\delta}^{2}-$ $B, a_{55}=2 \sigma_{\epsilon}^{4}+\beta^{2} B$.

The above results clearly show that the variances and covariances of the estimated parameters converge to 0 as $n$ tends to infinity. Hence all parameters are consistent.

\section{SIMULATION STUDY}

A simulation study was carried out to support the algebraic results presented earlier. For simulation experiments, we considered the various parameter settings given in Table 1 . In selecting $\sigma_{X}^{2}$ we follow the principle of Ref. 8 in which the difference caused by measurement errors will probably be dominated by the difference between the mean levels. For each specified set of parameter values, 15000 simulated data sets were obtained for the sample sizes $n$ in Table 1. The performance of the estimated parameters are measured by estimated bias (EB), estimated root mean square error (ERMSE) and standard deviation $(\mathrm{SD})$ where $\mathrm{EB}=|\overline{\hat{w}}-w|$ and ERMSE $=$ $\sqrt{(1 / s) \sum\left(\hat{w}_{j}-w\right)^{2}}$ where $w$ is a generic term for 
Table 1 Results for $\alpha=0, \mu=10, \sigma_{X}^{2}=5$ with 3 different sets of $\left(\beta, \sigma_{\delta}, \sigma_{\epsilon}\right) . n$ is the sample size.

\begin{tabular}{|c|c|c|c|c|c|c|c|c|c|c|c|c|c|c|c|c|}
\hline \multirow[t]{2}{*}{ Statistic } & \multirow[t]{2}{*}{$n$} & \multicolumn{5}{|c|}{$0.80,0.90,0.7(5)$} & \multicolumn{5}{|c|}{$(1.00,0.75,1.00)$} & \multicolumn{5}{|c|}{$(1.20,1.00,0.80)$} \\
\hline & & $100 \mu$ & $\alpha$ & $\beta$ & $\sigma_{X}^{2}$ & $\sigma_{\delta}^{2}$ & $100 \mu$ & $\alpha$ & $\beta$ & $\sigma_{X}^{2}$ & $\sigma_{\delta}^{2}$ & $100 \mu$ & $\alpha$ & $\beta$ & $\sigma_{X}^{2}$ & $\sigma_{\delta}^{2}$ \\
\hline \multirow[t]{6}{*}{ EB } & 50 & 0.102 & 0.0033 & 0.098 & 0.0128 & 0.0184 & 0.150 & 0.0020 & 0.110 & 0.0157 & 0.0239 & 0.186 & 0.0017 & 0.105 & 0.0182 & 0.0215 \\
\hline & 70 & 551 & 0.0024 & 0.056 & 0.0112 & 0132 & 0.092 & 0.0015 & 0.082 & 0.0120 & 0.0153 & 0.041 & 0.0012 & 0.074 & 0.0166 & 0.0142 \\
\hline & 100 & 0.021 & 0.0005 & 0.048 & 0.0082 & 0.0075 & 0.030 & 0.0011 & 0.048 & 0.0057 & 0.0138 & 0.036 & 0.0010 & 0.043 & 0.0065 & 0.0088 \\
\hline & 200 & 0.011 & 0.0003 & 0.028 & 0.0068 & 0.0042 & 0.025 & 0.0008 & 0.024 & 0.0045 & 0.0079 & 0.024 & 0.0008 & 0.018 & 0.0063 & 0.0060 \\
\hline & 300 & 0.004 & 0.0001 & 0.014 & 0.0043 & 0.0027 & 0.015 & 0.0006 & 0.016 & 0.0031 & 0.0024 & 0.002 & 0.0006 & 0.013 & 0.0058 & 0.0045 \\
\hline & 500 & 0.002 & 0.0000 & 0.003 & 0.0017 & 0.0011 & 0.001 & 0.0001 & 0.012 & 0.0010 & 0.0016 & 0.001 & 0.0002 & 0.007 & 0.0002 & 0.0029 \\
\hline \multirow[t]{6}{*}{ ERMSE } & 50 & 16.322 & 0.3441 & 1.108 & 0.5054 & 0.3378 & 18.742 & 0.3416 & 1.091 & 0.4530 & 0.4711 & 21.090 & 0.3440 & 1.086 & 0.4468 & 0.6051 \\
\hline & 70 & 13.843 & 0.2890 & 0.920 & 0.4249 & 0.2849 & 15.560 & 0.2864 & 0.921 & 0.3847 & 0.3971 & 17.821 & 0.2935 & 0.912 & 0.3836 & 0.5110 \\
\hline & 100 & 11.524 & 0.2439 & 0.771 & 0.3654 & 0.2421 & 13.171 & 0.2411 & 0.775 & 0.3298 & 0.3408 & 14.815 & 0.2420 & 0.765 & 0.3193 & 0.4271 \\
\hline & 200 & 9.301 & 0.0813 & 0.548 & 0.2557 & 0.1715 & 10.921 & 0.1240 & 0.542 & 0.2294 & 0.2365 & 11.303 & 0.1864 & 0.546 & 0.2246 & 0.3020 \\
\hline & 300 & 5.783 & 0.0667 & 0.449 & 0.2057 & 0.1387 & 8.799 & 0.0760 & 0.451 & 0.1868 & 0.1 & & 0.1270 & 0.438 & 0.1857 & 0.2489 \\
\hline & 500 & 0.930 & 0.0515 & 0.352 & 0.1599 & 0.1065 & 3.803 & 0.0586 & 0.344 & 0.1453 & 0.1512 & 3.955 & 0.0868 & 0.341 & 0.1438 & 0.1934 \\
\hline \multirow[t]{6}{*}{$\mathrm{SD}$} & 50 & 16.129 & 0.3402 & 1.086 & 0.5008 & 0.3335 & 18.493 & 0.3353 & 1.067 & 0.4507 & 0.4687 & 20.932 & 0.3428 & 1.057 & 0.4451 & 0.5960 \\
\hline & 70 & 13.663 & 0.2887 & 0.926 & 0.4262 & 0.2839 & & 0.2842 & 0.907 & 0.3833 & & & 0.2905 & 0.8 & & 0.5074 \\
\hline & 100 & 11.457 & 0.2417 & 0.776 & 0.3575 & 0.2384 & 13.156 & 0.2386 & 0.764 & 0.3226 & 0.3353 & 14.882 & 0.2439 & 0.757 & 0.3185 & 0.4263 \\
\hline & 200 & 10.021 & 0.1811 & 0.551 & 0.2536 & 0.1694 & 6.922 & 0.1233 & 0.543 & 0.2295 & 0.2385 & 9.082 & 0.1555 & 0.538 & 0.2262 & 0.3034 \\
\hline & 300 & 9.810 & 0.1064 & 0.451 & 0.2079 & 0.1387 & 4.825 & 0.0762 & 0.444 & 0.1877 & 0.1952 & 6.117 & 0.0962 & 0.439 & 849 & 0.2480 \\
\hline & 500 & 5.601 & 0.0814 & 0.350 & 0.1611 & 0.10 & 2314 & 0.0356 & 0.344 & 0.1353 & 0.1 & 1.257 & 0.0468 & 0.341 & 35 & 0.1623 \\
\hline \multirow[t]{6}{*}{$\mathrm{RM}$} & 50 & 1.663 & 0.0192 & 1.576 & 1.4605 & 0.7183 & 0.367 & 0.0024 & 1.955 & 1.8511 & 1.0099 & 1.277 & 0.0031 & 2.398 & 1.0024 & 2.7959 \\
\hline & 70 & 4.992 & 0.0051 & 1.414 & 0.8570 & 0.6991 & 1.224 & 0.0102 & 2.524 & 1.0834 & 0.7976 & 1.343 & 0.0279 & 2.109 & 0.5985 & 2.6316 \\
\hline & 100 & 0.905 & 0.0111 & 1.490 & 0.8325 & 0.6069 & 2.022 & 0.0010 & 1.383 & 1.0844 & 0.5668 & 1.100 & 0.0046 & 1.183 & 0.6714 & 1.2388 \\
\hline & 200 & 4.950 & 0.0826 & 1.651 & 0.5988 & & 5.575 & 0.0009 & 0.745 & 0.4 & & 10.744 & 0.1996 & 1.673 & 0.6524 & 0.4864 \\
\hline & 300 & 17.977 & 0.0947 & 0.909 & 0.6316 & 0.3417 & 3.701 & 0.0150 & 1.423 & 0.2456 & 0.7808 & 2.811 & 0.0054 & 1.302 & 0.7127 & 0.4937 \\
\hline & 500 & 19.141 & 0.1091 & 1.317 & 0.2878 & 0.2654 & 6.614 & 0.2964 & 0.924 & 0.3019 & 0.1103 & 3.253 & 0.0575 & 2.345 & 0.3223 & 0.2110 \\
\hline
\end{tabular}

the parameters and SD for each parameter are computed from the diagonal element of the asymptotic variance-covariance matrix. We also tried to investigate whether the distribution of the estimators are asymptotically normal or not. For testing normality of the distribution of the estimators we have used the rescaled moment $(\mathrm{RM})$ test proposed by Imon ${ }^{14}$ which is an extension of the Jarque-Bera normality test. We have used the RM test here because the Jarque-Bera test has a well known disadvantage of possessing poor power. When we denote the sample skewness and sample kurtosis of the estimators by $S$ and $K$, respectively, then the RM value is defined as

$$
\mathrm{RM}=\frac{n c^{2}}{6}\left[S^{2}+\frac{(K-3)^{2}}{4}\right]
$$

where $n$ is the sample size and $c=n /(n-k)$ when $k$ is the number of parameters. The distribution of RM follows a $\chi^{2}$ distribution with 2 degrees of freedom.

For each specified set of parameters in Table 1, the estimated bias (EB) of all parameters are close to 0 and become smaller when the sample size increases, which shows the unbiasedness of the parameters. From Table 1, the relative EB for $\sigma_{X}^{2}$ is 1.966 for $n=50$ and and it reduces to 0.058 for $n=500$. Similarly, the relative EB for $\sigma_{\delta}^{2}$ decreases from 1.422 for $n=50$ to 0.189 for $n=500$. Also, the decrease in relative $\mathrm{EB}$ for $\sigma_{\epsilon}^{2}$ is seen from 2.453 for $n=50$ to 0.147 for $n=500$. Similar remarks may apply with other measurements such as ERMSE and SD where these values tend to decrease with increase in sample sizes. These results clearly suggest that the estimated values of parameters are consistent. We also observe that each of the estimated parameters individually follows a normal distribution. Here, the cut-off value for the RM statistic is 5.99 but all RM values we obtain from this experiment are much less than this cut-off value.

\section{NUMERICAL EXAMPLE}

In this section we consider a real world data to estimate the parameter of this model. In order to make the relationship as model (1), we assume that measurement error can occur in both the dependent and independent variables of this data set.

Here we consider the serum kanamycin data taken from Kelly ${ }^{15}$. In order to measure the serum kanamycin levels in blood samples, simultaneous pairs of measurements were taken from twenty premature babies. These two measurements were obtained by the heel stick method (independent variable) and the umbilical catheter method (dependent variable).

We consider five different cases to estimate parameters and their standard deviation for serum kanamycin data and compared those with the results obtained by the proposed method. It is worth mentioning that case 4 is computationally impossible for any real data. The results are given in Table 2

Results presented in Table 2 give a clear indication of using the proposed method for estimating parameters and their standard deviations in a LSRM. Case 4 is impossible to compute. Out of 6 parameters we cannot estimate two parameters for case 1 and one parameter for case 2 , case 3 , and case 5 . Only the proposed method can estimate all 6 parameters. In terms of precision we observe that for $\mu$ our proposed 
Table 2 Comparison of estimated parameters (standard deviations in parentheses) of different MLE methods for serum kanamycin data.

\begin{tabular}{ccccccc}
\hline Case & $\mu$ & $\alpha$ & $\beta$ & $\sigma_{X}^{2}$ & $\sigma_{\delta}^{2}$ & $\sigma_{\epsilon}^{2}$ \\
\hline 1 & $20.9(1.5)$ & $-1.2(1.1)$ & $1.1(0.1)$ & $21.9(6.9)$ & - & - \\
2 & $20.9(1.1)$ & $2.0(7.3)$ & $0.9(0.2)$ & $23.7(7.8)$ & - & $26.7(8.7)$ \\
3 & $20.9(1.1)$ & $-4.4(274.9)$ & $1.2(4.5)$ & $0.9(282.9)$ & $23.9(7.8)$ & - \\
5 & $20.9(1.1)$ & $-1.2(3.3)$ & $1.1(0.2)$ & $20.4(7.8)$ & $4.4(1.4)$ & - \\
Proposed & $20.9(1.1)$ & $-0.1(0.7)$ & 1.0 & $21.3(7.5)$ & $3.4(3.4)$ & $5.4(3.7)$ \\
\hline
\end{tabular}

method has better precision than case 1 , however it has the same precision as cases 2, 3, and 5. For $\alpha$ the proposed method outperforms all four cases. This method yields lower standard deviations than all other cases. For $\sigma_{X}^{2}$ the proposed method gives smaller standard deviation than cases 2, 3, and 5 however its standard deviation is slightly more than that of case 1 . For $\sigma_{\delta}^{2}$ and $\sigma_{\epsilon}^{2}$ the proposed method clearly outperforms the other estimation methods. Thus the proposed method performs best overall.

\section{CONCLUSIONS}

In this article, a new maximum likelihood method is proposed to estimate parameters of a linear structural relationship model based on the assumption of known slope. We then derive the asymptotic variancecovariance matrix of the parameters in simple form. With the help of simulation study we have showed that the estimated values of the parameters are unbiased and consistent even for relatively small samples. Real world data shows that the proposed method has better precisions in comparison with the existing MLE alternatives.

Acknowledgements: The authors thank the referees and editorial staff for their very helpful comments and suggestions and the University of Malaya (RG239-12AFR) for their funding.

\section{REFERENCES}

1. Birch MW (1964) A note on the maximum likelihood estimation of a linear structural relationship. J Am Stat Assoc 59, 1175-8.

2. Barnett VD (1967) A note on linear structural relationships when both residual error variances are known. Biometrika 54, 670-2.

3. Madansky A (1959) The fitting of straight lines when both variables are subject to error. J Am Stat Assoc 54, 173-205.

4. Fuller WA (1987) Measurement Error Models, Wiley, New York.

5. Bolfarine H, Cordani LK (1993) Estimation of a structural linear regression model with known reliability ratio. Ann Inst Stat Math 45, 531-40.
6. Patefield WM (1985) Information from the maximized likelihood function. Biometrika 72, 664-8.

7. Wong MY (1989) Likelihood estimation of a simple linear regression model when both variables have error. Biometrika 76, 141-8.

8. Hood K, Barry AJ, Terence C (1999) Asymptotic information and variance-covariance matrices for the linear structural model. J R Stat Soc D 48, 477-93.

9. Dent B (1935) On observations of points connected by a linear relation. Proc Phys Soc 47, 92-106.

10. Housnerand GW, Brennan JF (1948) Estimation of linear trends. Ann Math Stat 19, 308-88.

11. Theil H (1950) A rank-invariant method of linear and polynomial regression analysis. Nederl Akad Wetensch Proc 53, 386-92.

12. Al-Nasser AD (2005) A new nonparametric method for estimating the slope of simple linear measurement error model in the presence of outliers. Pakistan J Stat 21, 265-74.

13. Kendall MG, Stuart A (1973) The Advanced Theory of Statistics, Griffin, London.

14. Imon AHMR (2003) Regression residuals, moments and their use in tests for normality. Comm Stat Theor Meth 32, 1021-34.

15. Kelly GE (1984) The influence function in the errors in variables problem. Ann Stat 12, 87-100. 